\title{
Cerebellar ataxia with neuropathy and bilateral vestibular areflexia syndrome (CANVAS): a histopathologic case report
}

\author{
David J Szmulewicz, \\ Department of Neuroscience, Alfred Hospital, Victoria, Australia
}

Saumil N Merchant, and

Massachusetts Eye and Ear Infirmary, and Harvard Medical School, Boston, Massachusetts, USA

G Michael Halmagyi

Department of Neurology, Royal Prince Alfred Hospital, New South Wales, Australia

Cerebellar ataxia with neuropathy and bilateral vestibular areflexia syndrome (CANVAS) represents a widespread impairment of the various systems involved in balance: cerebellar, proprioceptive and vestibular1. The characteristic clinical sign of this syndrome is the impaired visually enhanced vestibulo-ocular reflex (VVOR), which represents combined impairment of the three compensatory eye movement reflexes, namely the vestibulo-ocular reflex (VOR), smooth pursuit (SP) and optokinetic reflex (OKR). From the neuro-otological viewpoint the remarkable feature is the severe loss of vestibular function with preservation of auditory function. It is not known whether the vestibular loss in CANVAS is of central (vestibular nucleus) or peripheral origin, and if peripheral, whether of sensory or of neural origin. We describe the otopathology in a case of CANVAS that demonstrated the vestibular loss to be the result of bilateral neuropathy of the vestibular (Scarpa's) ganglion and nerve.

\section{CASE REPORT}

A 71 year old woman presented with a 10 year history of progressive imbalance, initially more noticeable in the dark. The imbalance progressed to needing a 4-wheel walker. There was no history of vertigo or aminoglycoside therapy, and no family history of imbalance. All 3 children ( 2 daughters and 1 son) were examined clinically.

On examination, there was severe bi-directional impairment of horizontal and vertical smooth pursuit and VVOR, and a positive bilateral head impulse test. Visual acuity dropped from $6 / 9$ to $6 / 36$ on vertical head shaking. There was marked cerebellar dysarthria, appendicular ataxia and a positive Romberg's test. The tendon reflexes, whilst normal in the upper limbs, were absent in the lower limbs. Proprioception was normal in the fingers and impaired in the toes. Pinprick sensation was normal.

Gene testing for SCA1, 2, 3, 6 and 7 and Friedreich's ataxia were negative, as were antibodies for Coeliac disease. MRI revealed cerebellar atrophy affecting the anterior and dorsal vermis with corresponding hemispheric atrophy predominantly affecting crus 12 . Audiogram demonstrated normal hearing for age. Caloric tests confirmed severe bilateral

Correspondence: David J Szmulewicz,dsz@me.com, Telephone: (+61) 390762552 Fax: (+61) 390762458.

This is a PDF file of an unedited manuscript that has been accepted for publication. As a service to our customers we are providing this early version of the manuscript. The manuscript will undergo copyediting, typesetting, and review of the resulting proof before it is published in its final citable form. Please note that during the production process errors may be discovered which could affect the content, and all legal disclaimers that apply to the journal pertain. 
impairment of lateral semicircular canal function. Sinusoidal testing confirmed SP, VOR and VVOR impairment (this patient's data can be seen in figure 1 of the original description3). Nerve conduction studies showed normal motor conduction but absent sensory action potentials in upper and lower limbs.

She died of inanition at age 85,14 years after symptom onset. Post-mortem examination of the brain revealed macroscopic cerebellar cortical atrophy of the anterior vermis and the supero-medial aspect of the cerebellar hemispheres. Microscopically there was diffuse Purkinje cell loss, principally affecting the vermis, but also evident in the lateral cerebellum. No brainstem abnormalities were noted. The sural nerve showed severe axonal neuropathy.

\section{Otopathology}

The otopathology was reviewed by two senior otopathologists who were blinded to the clinical details, and each has greater than 25 years experience in the field and over 150 peerreviewed original articles in otopathology. Both temporal bones showed similar findings and are described together. The external auditory canals, tympanic membranes, ossicles, tympanic cavities, mastoids and otic capsules appeared normal. The labyrinths were fully developed and both cochleae showed the normal 2-1/2 turns without endolymphatic hydrops. The organs of Corti appeared normal throughout, except in the extreme apex where there was a partial loss of outer hair cells. The striae vasculares showed patchy atrophy but were generally intact. The cochlear neuronal population appeared normal (for age) in both ears.

Within the vestibular systems, the most striking change was severe atrophy of both vestibular nerves (Figure 1a and b). There was a severe diminution of the number of Scarpa's ganglion cells on both sides with atrophy of peripheral and central axons. The total Scarpa's ganglion cell count (right, 2,596; left, 2,587) constituted a loss of $84 \%$ when compared to the predicted normal mean count for age 4 . Notably, the vestibular sense organs (cristae and maculae) in both ears showed a good population of hair cells and supporting cells (Figure 1c).

The facial nerves showed atrophy, especially affecting the geniculate ganglia (Figure 1d). A significant portion of the trigeminal ganglion was present within the specimen in the left temporal bone and showed severe atrophy with a marked decrease in ganglion cells and atrophy of nerve fibers.

\section{DISCUSSION}

Our patient had a selective sensory ganglionopathy affecting the vestibular, facial and trigeminal nerves, but remarkably sparing the cochlear nerves and its (spiral) ganglia, a finding not previously reported. We do not have adequate clinical data regarding function of the trigeminal or facial nerve, in the several years preceding the patient's demise, which may explain the discrepancy between the clinical presentation and the histopathology. Spinal sensory ganglionopathies occur with paraneoplastic disease, immune disorders (Sjögren's syndrome, Miller Fisher syndrome and Bickerstaff's brainstem encephalitis), drugs (cisplatin and pyridoxine) and inherited disorders (Friedreich's ataxia and SCA 4). In our experience, CANVAS patients have uniformly tested negative for all of these. In a subset of patients with chronic ataxic neuropathy, none of these associations are identified, which generally results in a diagnosis of chronic idiopathic ataxic neuropathy5. CANVAS could account for some of these.

This first insight into the otopathology in CANVAS reveals marked neural atrophy (indicative of a degenerative process), particularly affecting the vestibular, trigeminal and 
facial ganglia but sparing the spiral (cochlear) ganglion. Further studies might help to elucidate the cause of this cranial sensory ganglionopathy.

\section{Acknowledgments}

Disclosure of funding: Supported by NIH grant U24 DC008559

\section{REFERENCES}

1. Szmulewicz, et al. Neuropathy: an integral component of cerebellar ataxia neuropathy vestibular areflexia syndrome. Neurology. Reviewed with a request for revision.

2. Schmahmann, JD.; Doyon, J.; Petrides, M.; Evans, et al. MRI Atlas of the Human Cerebellum. Academic Press; San Diego: 2000.

3. Migliaccio AA, Halmagyi GM, McGarvie LA, et al. Cerebellar ataxia with bilateral vestibulopathy: description of a syndrome and its characteristic clinical sign. Brain. 2004; 127:280-293. [PubMed: 14607788]

4. Merchant, SN.; Nadol, JB. Schuknecht's Pathology of the Ear. 3rd edition. PMPH-USA Inc; Shelton, CT: 2010.

5. Kuntzer, et al. Clinical features and pathophysiological basis of sensory neuronopathies (ganglionopathies). Muscle Nerve. 2004; 30:255-268. [PubMed: 15318336] 


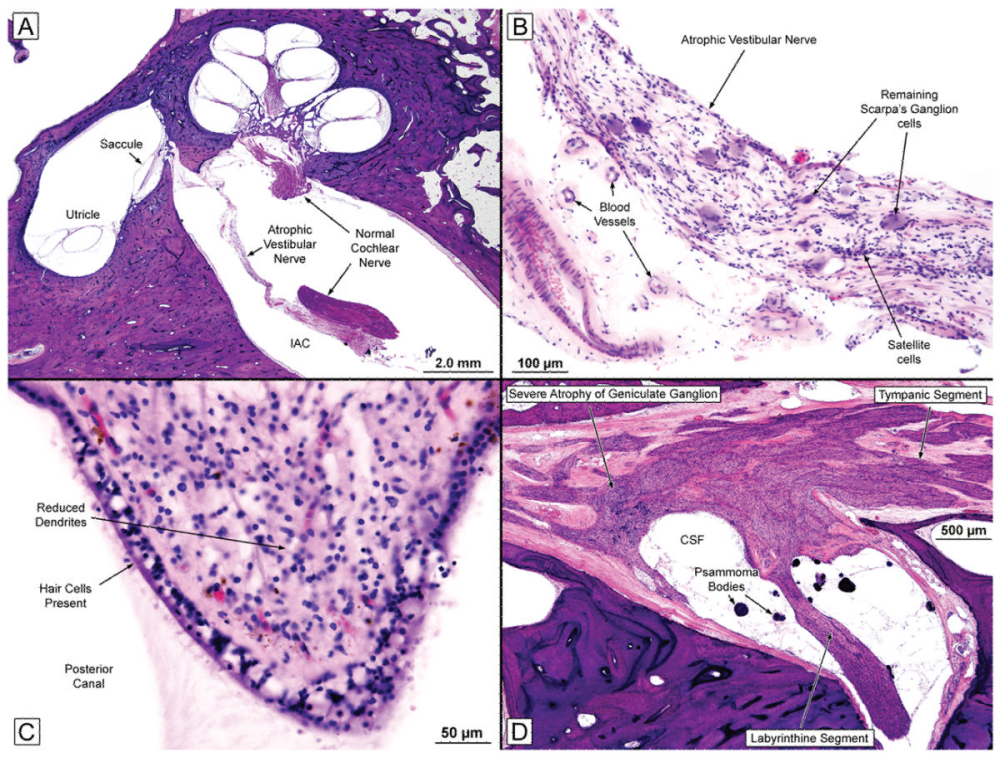

FIG. 1.

$A$, Low-power view of the left ear (near-mid modiolar section) showing an atrophic vestibular nerve and a normal-appearing cochlear nerve. The cochlear turns, saccule, and utricle seemed normal.

$B$, High-power view of the left vestibular nerve within the internal auditory canal. The nerve was severely atrophic, but there was no evidence of inflammation or vasculitis. There was severe loss of Scarpa's ganglion cells.

$C$, Crista of the left posterior semicircular canal showing a good population of hair cells and supporting cells in the neuroepithelium. There was a reduction of dendrites within the stroma.

$D$, Facial nerve from the right ear. There was severe diminution of the number of geniculate ganglion cells. CSF indicates cerebrospinal fluid. 